\title{
ARTICLE
}

Clinical Study

\section{Randomised phase II trial of mFOLFOX6 plus bevacizumab versus mFOLFOX6 plus cetuximab as first-line treatment for colorectal liver metastasis (ATOM trial)}

Eiji Oki $\mathbb{D}^{1}$, Yasunori Emi ${ }^{2}$, Takeharu Yamanaka ${ }^{3}$, Hiroyuki Uetake ${ }^{4}$, Kei Muro ${ }^{5}$, Takao Takahashi ${ }^{6}$, Takeshi Nagasaka ${ }^{7}$, Etsuro Hatano ${ }^{8}$, Hitoshi Ojima $^{9}$, Dai Manaka ${ }^{10}$, Tetsuya Kusumoto ${ }^{11}$, Yu Katayose ${ }^{12}$, Toshiyoshi Fujiwara ${ }^{13}$, Kazuhiro Yoshida ${ }^{6}$, Michiaki Unno ${ }^{14}$, Ichinosuke Hyodo ${ }^{15}$, Naohiro Tomita ${ }^{16}$, Kenichi Sugihara ${ }^{4}$ and Yoshihiko Maehara ${ }^{17}$

BACKGROUND: Chemotherapy with biologics followed by liver surgery improves the resection rate and survival of patients with colorectal liver metastasis (CRLM). However, no prospective study has compared the outcomes of chemotherapy with bevacizumab (BEV) versus cetuximab (CET).

METHODS: The ATOM study is the first randomised trial comparing BEV and CET for initially unresectable CRLM. Patients were randomly assigned in a 1:1 ratio to receive mFOLFOX6 plus either BEV or CET. The primary endpoint was progression-free survival (PFS).

RESULTS: Between May 2013 and April 2016, 122 patients were enrolled. Median PFS was 11.5 months (95\% Cl 9.2-13.3 months) in the BEV group and 14.8 months ( $95 \% \mathrm{Cl} 9.7-17.3$ months) in the CET group (hazard ratio $0.803 ; P=0.33$ ). Patients with a smallernumber but larger-sized metastases did better in the CET group. In the BEV and CET groups, the response rates were $68.4 \%$ and $84.7 \%$ and the resection rates were $56.1 \%$ and $49.2 \%$, respectively.

CONCLUSION: Although CET achieved a better response rate than BEV for patients with a small number of large liver metastases, both biologics had similar efficacy regarding liver resection and acceptable safety profiles. To achieve optimal PFS, biologics should be selected in accordance with patient conditions.

TRIAL REGISTRATION: This trial is registered at ClinicalTrials.gov (number NCT01836653), and UMIN Clinical Trials Registry (UMIN-CTR number UMIN000010209).

British Journal of Cancer (2019) 121:222-229; https://doi.org/10.1038/s41416-019-0518-2

\section{BACKGROUND}

Colorectal cancer (CRC) is the third most common cancer and the second leading cause of cancer death worldwide. ${ }^{1}$ The 5 -year overall survival (OS) rate for the curatively resectable stages I-III of CRC is almost $80 \%$ but is only $13 \%$ for stage IV CRC, which accounts for approximately $18 \%$ of CRC diagnoses. ${ }^{2}$ Liver metastases develop in almost $60 \%$ of patients with stage IV CRC and in $9-13 \%$ of patients after curative resection of CRC. ${ }^{1,3}$ To improve the prognosis of patients with CRC, outcomes for patients with liver metastasis must be improved.

Among the treatment options for colorectal liver metastasis (CRLM), liver resection is the most conducive to a cure, with 5-year
OS rates of $29-48 \%$.,5 Even for initially unresectable liver metastasis, effective chemotherapy, along with targeted therapy, sometimes enables resection of liver metastasis; Adam et al. reported a 5 -year OS rate of $33 \%$ for patients with unresectable metastases who underwent surgery after chemotherapy. ${ }^{4}$ However, it is unclear which type of chemotherapy can best increase resection rates and improve survival in patients with CRLM.

Several promising CRLM treatments have been reported, including chemotherapy and molecular agents that target epidermal growth factor receptor (EGFR) and vascular endothelial growth factor (VEGF). ${ }^{6-10}$ Anti-EGFR drugs resulted in high response and resection rates in the CELIM phase II trial and other

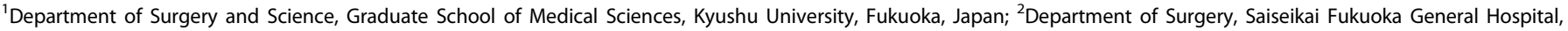

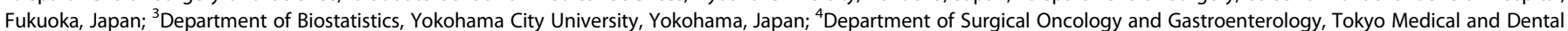

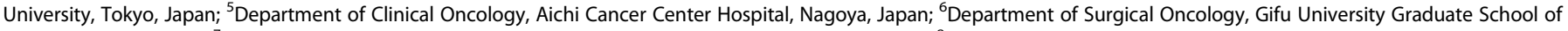

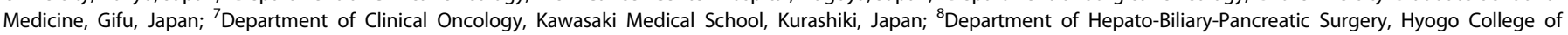

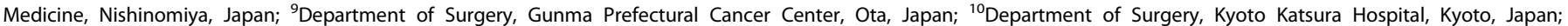

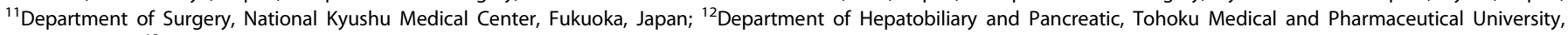

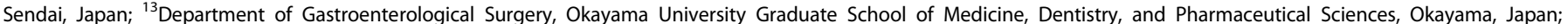

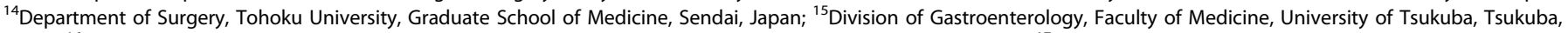

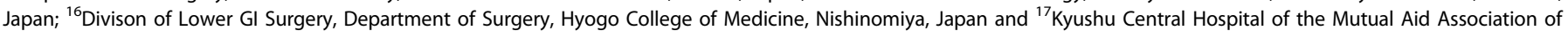
Public School Teachers, Fukuoka, Japan

Correspondence: Eiji Oki (okieiji@surg2.med.kyushu-u.ac.jp)

Received: 16 March 2019 Revised: 12 June 2019 Accepted: 20 June 2019

Published online: 9 July 2019 
studies for initially unresectable CRLM with wild-type $K R A S^{7,8,11}$ Anti-VEGF antibody regimens, such as mFOLFOX6 or CAPEOX plus bevacizumab (BEV), have also showed high response and resection rates in phase II studies. ${ }^{9,10,12}$ These reports suggest that the combination of targeted agents and chemotherapy can increase liver resection rates and response rates, thus improving the progression-free survival (PFS) and OS of patients with CRLM. However, no studies have compared anti-VEGF agents and antiEGFR agents for liver-limited $R A S^{w t}$ CRLM.

The present randomised phase II clinical study (the ATOM trial) aimed to compare mFOLFOX6 plus BEV versus mFOLFOX6 plus cetuximab (CET) in patients with liver-limited $R A S^{\text {wt }}$ CRLM.

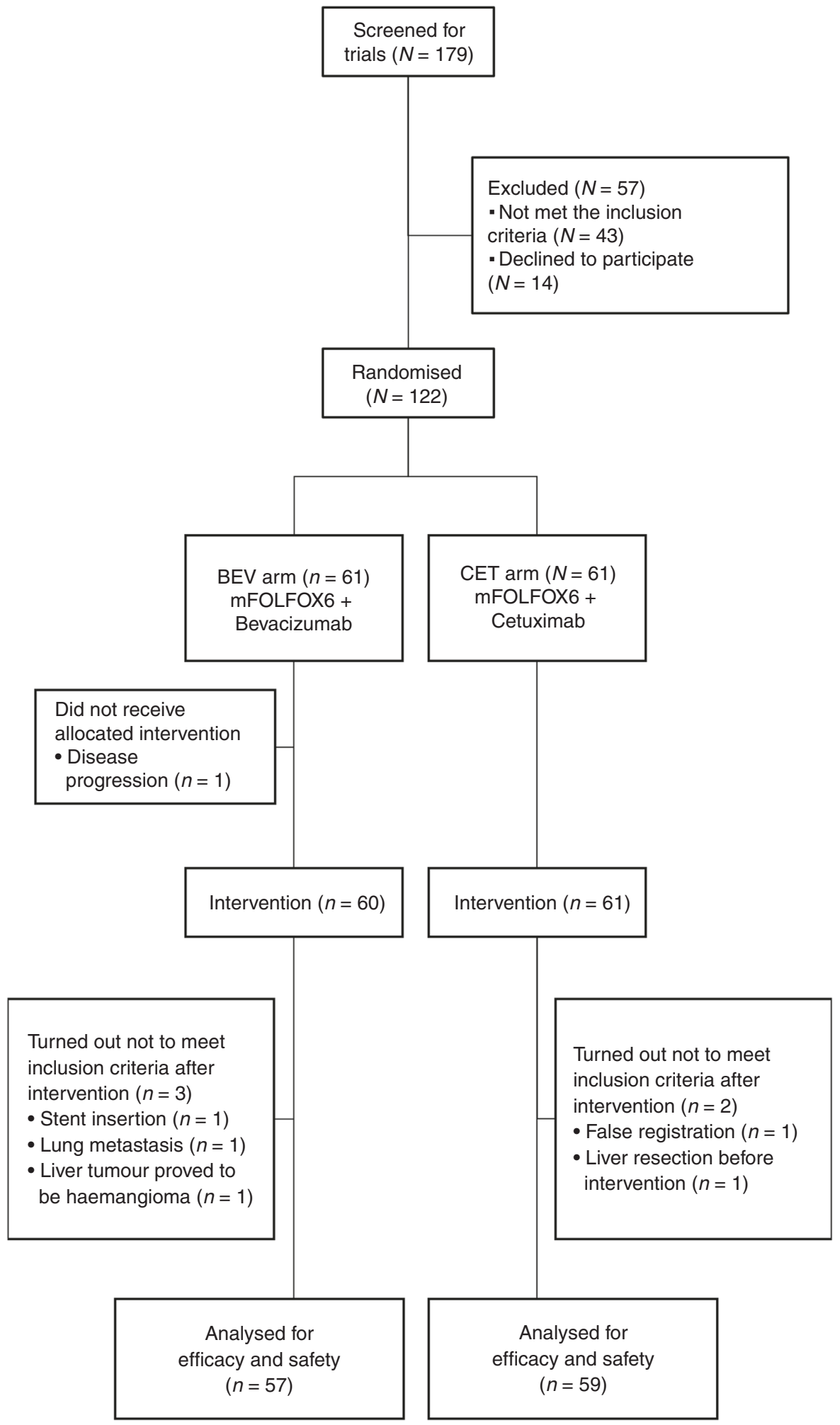

Fig. 1 Consort flow diagram. Primary analysis was evaluated in the full analysis set (FAS), which was defined as all patients who were eligible for study inclusion, as well as those who received at least one dose of the protocol treatment 


\section{METHODS}

Patients

The ATOM trial was a multicentre, randomised phase II study designed to evaluate the efficacy and safety of mFOLFOX6 plus BEV and mFOLFOX6 plus CET in patients with liver-limited metastases from wild-type (K)RAS CRC. Eligible criteria for the study were (1) histopathologically confirmed CRC (adenocarcinoma), excluding appendix and anal cancers; (2) no metastasis other than to liver; (3) tumour tested to be KRASwt (between May 2013 and April 2015) or RASwt (between April 2015 and April 2016) (4) age between 20 and 80 years at the time of enrolment; (5) Eastern Cooperative Oncology Group performance status of 0 or 1 ; (6) life expectancy of $\geq 3$ months at the time of enrolment; (7) sufficient organ function; and (8) signed informed consent. Furthermore, each patient had to satisfy at least one of the following criteria at enrolment: (a) $\geq 5$ liver metastases, (b) a liver metastasis with a maximum diameter $\geq 5 \mathrm{~cm}$, (c) technically inappropriate for resection in light of remaining hepatic function, (d) invasion into all hepatic veins or the inferior vena cava, or (e) invasion into both the right and left hepatic arteries or both of the portal veins. The study was approved by the local ethics committee of each participating centre and conducted in 63 Japanese institution.

\section{Randomisation}

Randomisation was based on dynamic allocation by a minimisation method in a centralised web-based system (EPS Corporation, Tokyo, Japan). Allocation factors included: (1) synchronous liver metastases with a primary lesion, synchronous liver metastases without a primary lesion, or metachronous liver metastases; (2) number of metastases (1-4 or $\geq 5$ ); (3) maximum metastasis diameter ( $\leq 5$ or $>5 \mathrm{~cm}$ ); and (4) oxaliplatin used as adjuvant chemotherapy.

\section{Procedures}

Patients received either mFOLFOX6 plus BEV (BEV $5 \mathrm{mg} / \mathrm{kg}$, followed by oxaliplatin $85 \mathrm{mg} / \mathrm{m}^{2}$, l-leucovorin $200 \mathrm{mg} / \mathrm{m}^{2}$ and bolus infusion of fluorouracil $400 \mathrm{mg} / \mathrm{m}^{2}$ on Day 1 and continuous fluorouracil infusion $2400 \mathrm{mg} / \mathrm{m}^{2}$ on Day 1 through Day 2) or $\mathrm{mFOLFOX6}$ plus CET (CET $400 \mathrm{mg} / \mathrm{m}^{2}$ as the initial dose and 250 $\mathrm{mg} / \mathrm{m}^{2}$ as the subsequent doses on Days 1 and 8 , followed by mFOLFOX6), no later than 2 weeks after enrolment in the study. Study treatments were continued in 2-week cycles until disease progression. In accordance with the Response Evaluation Criteria In Solid Tumours version 1.1, the same methods were used to perform tumour assessment at baseline and every subsequent 8 weeks using torso contrast-enhanced computed tomography (CT), liver contrast-enhanced magnetic resonance imaging, or whole-body non-contrast CT. The tumour histopathological response rate was defined as the proportion of patients with grade $\geq \mathrm{lb}$ in accordance with the following definition: grade 0 , no necrosis in the tumour; grade $1 a$, necrosis in $<33.3 \%$ of the tumour; grade $1 \mathrm{~b}$, necrosis in $33.3-66.6 \%$ of the tumour; grade 2 , necrosis in $66.6-<100 \%$ of the tumour; and grade 3 , necrosis in $100 \%$ of the tumour.

Patients underwent liver resections if their metastases were considered resectable, based on tumour assessments performed after receiving at least 8 cycles of either protocol treatment. After liver resection, a total of 12 cycles of the same chemotherapy plus biologic agent as the preoperative treatment were recommended. Surgery was performed at least 42 days after the last dose of BEV.

Statistical analysis

The primary endpoint was PFS, as assessed by the Independent Central Review Committee (IRC). The cases were not censored at the time of liver resection. Recurrence was considered to be a PFS event in patients who underwent liver resection after protocol treatment. Secondary endpoints included response rate, tumour

\begin{tabular}{|c|c|c|c|c|}
\hline Variables & & $\begin{array}{l}\text { mFOLFOX6 } \\
+ \text { BEV }\end{array}$ & $\begin{array}{l}\text { mFOLFOX6 } \\
+ \text { CET }\end{array}$ & $P$ \\
\hline Age (years) & Median (range) & $64(32.0-80.0)$ & $65(42.0-79.0)$ & 0.328 \\
\hline \multirow[t]{2}{*}{ Sex } & Male & 34 (59.6\%) & 34 (57.6\%) & 0.825 \\
\hline & Female & 23 (40.4\%) & 25 (42.4\%) & \\
\hline \multirow[t]{2}{*}{ ECOG PS } & 0 & 51 (89.5\%) & $51(86.4 \%)$ & 0.616 \\
\hline & 1 & $6(10.5 \%)$ & $8(13.6 \%)$ & \\
\hline $\begin{array}{l}\text { Adjuvant } \\
\text { chemotherapy }\end{array}$ & Yes & $3(5.3 \%)$ & $2(3.4 \%)$ & 0.619 \\
\hline Prior oxaliplatin & Yes & $1(1.8 \%)$ & 2 (3.4\%) & 0.579 \\
\hline \multirow{2}{*}{$\begin{array}{l}\text { Location } \\
\text { of tumour }\end{array}$} & Right & $9(15.8 \%)$ & $14(23.7 \%)$ & 0.284 \\
\hline & Left & 48 (84.2\%) & 45 (76.3\%) & \\
\hline \multirow[t]{3}{*}{ Tumour status } & $\begin{array}{l}\text { Synchro/ } \\
\text { primary tumour }\end{array}$ & 13 (22.8\%) & $15(25.4 \%)$ & 0.771 \\
\hline & $\begin{array}{l}\text { Synchro/no } \\
\text { primary } \\
\text { tumour }\end{array}$ & 39 (68.4\%) & 39 (66.1\%) & \\
\hline & Metachronous & 5 (8.8\%) & 5 (8.5\%) & \\
\hline \multirow{2}{*}{$\begin{array}{l}\text { Number of liver } \\
\text { metastases }\end{array}$} & $<5$ & 15 (26.3\%) & $18(30.5 \%)$ & 0.617 \\
\hline & $\geq 5$ & 42 (73.7\%) & $41(69.5 \%)$ & \\
\hline \multirow[t]{2}{*}{$\begin{array}{l}\text { Diameter of } \\
\text { liver metastases }\end{array}$} & $\leq 5 \mathrm{~cm}$ & 20 (35.1\%) & 19 (32.2\%) & 0.742 \\
\hline & $>5 \mathrm{~cm}$ & 37 (64.9\%) & $40(67.8 \%)$ & \\
\hline
\end{tabular}

mFOLFOX6 5-fluorouracil/folinic acid, oxaliplatin, BEV bevacizumab, CET cetuximab, ECOG PS Eastern Cooperative Oncology Group performance status

${ }^{\mathrm{a} S y n c h r o / n o}$ primary tumour: metastatic tumour diagnosed within 6 months after resection of primary tumour

shrinkage at week 8 , liver resection rate, time to treatment failure, OS, quality of life, and adverse events (AEs).

We employed a selection design based on the hazard ratio (HR). For 1 -year PFS rates of the two arms to be $55 \%$ and $50 \%$ (which corresponds to a HR of 0.862 under exponential distribution), we needed 160 patients to select better treatment in terms of an HR with a probability of $75 \%$. Therefore, the initial sample size was 160 in both arms. However, owing to slow accrual of patients, the independent data monitoring committee approved a reduction of the sample size in January 2015 to 120 patients in both arms; this would enable the observation of 84 PFS events, which would indicate a superior treatment with a probability of $70 \%$.

HRs for progression or death for CET versus BEV were estimated using a Cox proportional-hazards model. Survival curves were estimated using the Kaplan-Meier method. Statistical analyses were performed using SAS version 9.4 (SAS Institute, Cary, USA).

\section{RESULTS}

Patients

Between May 2013 and April 2016, we screened 179 patients and enrolled 122 patients from 63 sites in Japan (61 patients in each arm). Figure 1 shows the consort flow diagram. Five patients (two CET arm/three BEV arm) did not meet the criteria and a patient in the BEV arm cannot be treated because of rapid disease progression. Patient characteristics were well balanced between the two arms (Table 1). Of the 116 patients, 2 patients in the BEV arm were found to have RAS mutations, while 1 patient in each arm did not undergo RAS testing due to insufficient tumour samples. 
a

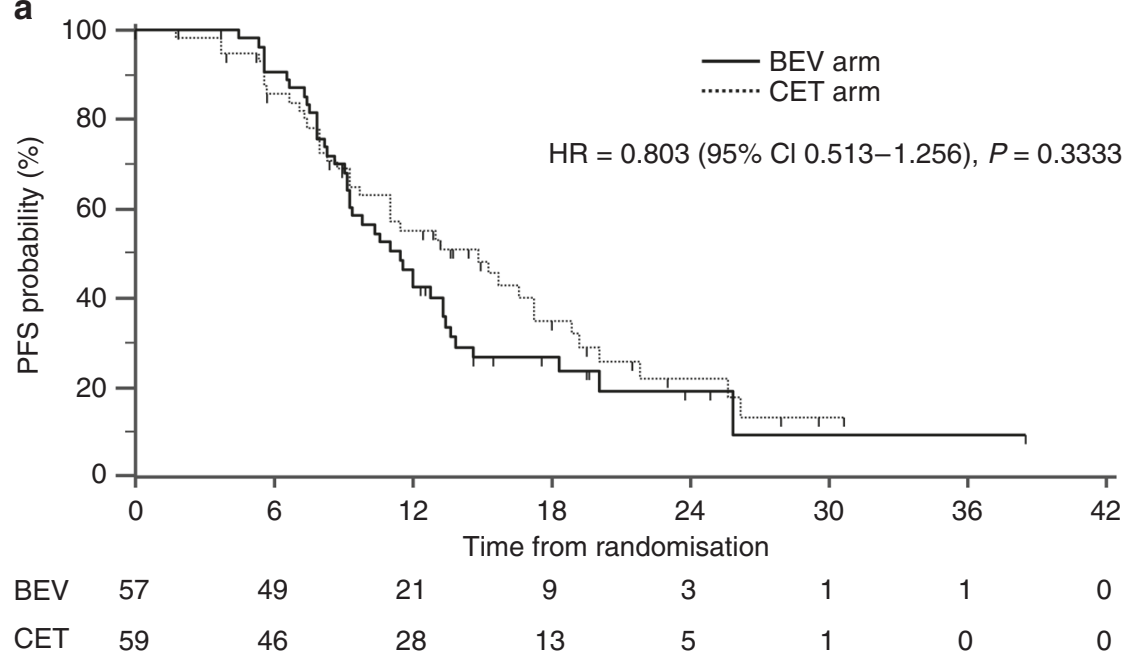

b

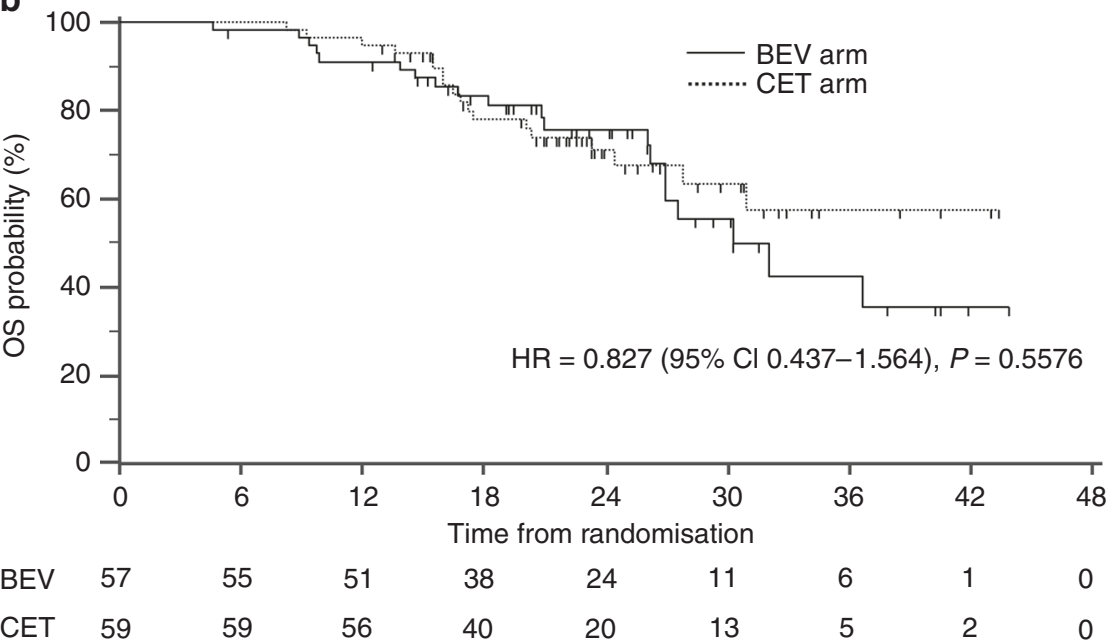

Fig. 2 Kaplan-Meier estimates of a progression-free survival by central assessment and b overall survival. Solid black line: mFOLFOX6 + bevacizumab, dotted black line: mFOLFOX6+cetuximab

Efficacy

On March 31, 2017, the median follow-up time was 24.3 months. The median PFS assessed by the IRC for the CET arm was 14.8 months ( $95 \%$ confidence interval $(\mathrm{Cl})$ : 9.7-17.3 months), while for the BEV arm it was 11.5 months (95\% Cl: 9.2-13.3 months), with a log-rank $P$ value of 0.33 . The PFS HR between the two arms was 0.803 (95\% Cl: 0.513-1.256; Fig. 2a); PFS in accordance with the investigators' evaluations is shown in Supplementary Fig. S1. The median OS in the BEV arm was 30.4 months but was not achieved yet in Cmab arm (HR: 0.827, 95\% Cl: 0.437-1.564; Fig. 2b). Subgroup analyses for the IRC PFS are shown in Fig. 3 Only in patient with 1-4 liver metastasis the CET arm showed significant $\mathrm{HR}$, whereas PFS was better in the BEV arm among patients with $\geq 5$ metastases. Kaplan-Meier curves of these subgroups are shown in Supplementary Fig. S2A, B.

The overall response rate (ORR) for BEV was $68.4 \%(95 \% \mathrm{Cl}$ : $54.8 \%-80.1 \%)$, while the ORR for CET was $84.7 \%(95 \% \mathrm{Cl}$ : 73.0\%-92.8\%; $P=0.0483$; Table 2). Median tumour shrinkage rates at 8 weeks were $25.3 \%$ for BEV and $37.8 \%$ for CET. The waterfall plot showing the best change in target lesion based on central assessment is shown in Fig. 4. The overall resection rates including R2 were $56.1 \%(32 / 57)$ for BEV and $49.2 \%$ (29/59) for CET, and resection rates (R0+R1) were $49.1 \%(28 / 57)$ for BEV and $47.5 \%$ (28/59) for CET (Table 2). Median PFS was assessed for patients with
R0/R1 status after study treatment followed by surgical resection; median PFS for BEV was 6.5 months (95\% Cl: 4.0-13.6), while that for CET it was 13.8 months (95\% Cl: 8.4-not reached), HR: 0.610 (95\% Cl: 0.298-1.245; Supplementary Fig. S3). Of the 57 tumours that were assessable for histopathological analysis, the tumour histopathological response rate (Grade $1 \mathrm{~b} / 2 / 3)$ was $66.6 \%$ (20/30) for BEV and $92.6 \%(25 / 27)$ for CET ( $P=0.0229$, Supplementary Table S1).

Safety

Grade $\geq 3$ subjective or objective toxicity events occurred in $40.4 \%$ of the patients who received BEV and $52.5 \%$ of the patients who received CET. The most frequently occurring $A E$ of grade $\geq 3$ was neutropenia, with an incidence of $36.8 \%$ in the BEV arm and $50.8 \%$ in the CET arm. Other AEs are presented in Supplementary Table S2. AEs that caused discontinuation occurred in 8 patients (13.1\%) in the BEV arm and $6(9.8 \%)$ in the CET arm. No patient died from treatment-related AEs. In the surgical safety population ( $n=33$ in the BEV arm and $n=29$ in the CET arm), all-grade surgery-related AEs according to the Clavien-Dindo classification were reported in 8 patients (24.2\%) in the BEV arm and $12(41.4 \%)$ in the CET arm. The most frequent surgical $A E$ was bile leakage, with an incidence of $18.2 \%$ in the BEV arm and $24.1 \%$ in the CET arm. No grade 5 AEs were reported (Supplementary Table S3). 


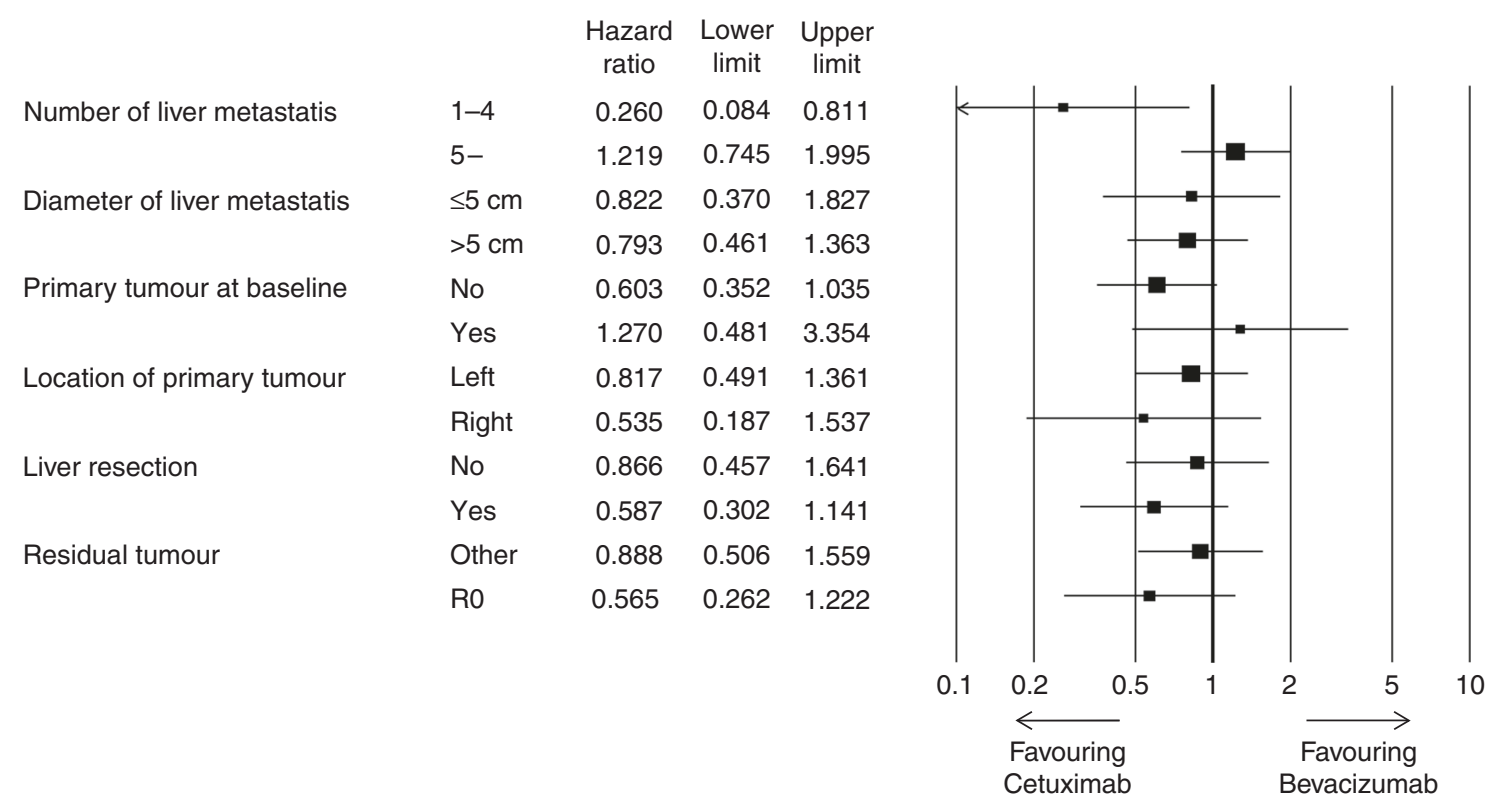

Fig. 3 Forest plots show hazard ratios for progression-free survival in patients with colorectal to liver metastases, using mFOLFOX6 + bevacizumab and mFOLFOX6+cetuximab

Table 2. Overall response rates and liver resection rate

\begin{tabular}{llll}
\hline & \multicolumn{2}{l}{ Overall response rates } & $P$ \\
\cline { 2 - 3 } & mFOLFOX6+BEV & mFOLFOX6+CET & \\
\hline CR (\%) & $1(1.8 \%)$ & $1(1.7 \%)$ & - \\
PR (\%) & $38(66.7 \%)$ & $49(83.1 \%)$ & - \\
SD (\%) & $18(31.6 \%)$ & $6(10.2 \%)$ & - \\
PD (\%) & $0(0.0 \%)$ & $2(3.4 \%)$ & - \\
NE & $0(0.0 \%)$ & $1(1.7 \%)$ & - \\
Overall response rate & $68.4 \%$ & $84.7 \%$ & 0.0483 \\
Disease control rate & $100 \%$ & $94.9 \%$ & 0.2437 \\
& & & \\
\hline & Liver resection rate & & \\
\cline { 2 - 3 } & mFOLFOX6+BEV & mFOLFOX6+CET \\
\hline R0+R1 & $28(49.1 \%)$ & $28(47.5 \%)$ & \\
R0 & $25(43.9 \%)$ & $22(37.3 \%)$ & \\
R1 & 3 (5.3\%) & $6(10.2 \%)$ & \\
R2 & $4(7.0 \%)$ & $1(1.7 \%)$ & \\
All & $32(56.1 \%)$ & $29(49.2 \%)$ & \\
Reasons for R2 & Ablation: 2 & Ablation and Residual: 1 \\
& Residual: 2 & & \\
\hline BEV & & & \\
\hline
\end{tabular}

BEV bevacizumab, CET cetuximab, CR complete response, mFOLFOX6 5fluorouracil/folinic acid, oxaliplatin, $N E$ not evaluable, $P D$ progressive disease, $P R$ partial response, $S D$ stable disease

\section{DISCUSSION}

The anti-VEGF antibody BEV has an anti-angiogenic effect, which elicits a specific morphologic tumour response of enhanced necrosis, thus increasing the survival benefit of chemotherapy. ${ }^{13}$ Because of these benefits, some pivotal clinical studies reported that combined therapy with cytotoxic drugs could potentially improve CRC prognosis. ${ }^{10}$ A phase II study of patients with CRLM reported that the anti-EGFR antibody CET was associated with an improved response rate and a high liver resection rate. ${ }^{7,14-17}$
Although a few head-to-head randomised studies compared the two antibodies in the whole population of patients with advanced $\mathrm{CRC}$, none of them led to a clear conclusion as to which agent was better for the liver-limited CRLM. ${ }^{15,17-19}$ Our trial aimed to evaluate the effect of an anti-EGFR antibody versus an anti-VEGF antibody in patients with CRLM.

In our analysis, the median PFS (as assessed by the IRC) tended to be better in the CET arm than in the BEV arm, but this difference was not statistically significant. The ORR was also better in the CET arm (84.7\%) than the BEV arm (68.4\%). However, despite the higher ORR in the CET arm compared with the BEV arm, the overall resection rate was similar in both arms. Although the main results were equivocal, we can consider the results from various angles.

Resection rate is often used as the primary endpoint for phase II studies evaluating chemotherapy for CRLM. Previous studies have reported similar resection rates to those achieved in our study. One study reported a liver resection rate and ORR of FOLFOXIRI + BEV of $51 \%$ and $81 \%$, respectively, ${ }^{10}$ while the CELIM trial reported that the resection rates of FOLFOX+CET and FOLFIRI + CET were $51 \%$ and $49 \%$, respectively. ${ }^{7}$ Furthermore, a review of data from previous studies reported that response and resection rates were positively correlated in CRLM and found the liver resection rate to be $50-60 \%$ when the ORR is $80 \% .^{5}$ This correlation is applicable to recent reports, including our study. However, increasing the response rate $>80 \%$ is a clinical challenge. Therefore, the resection rate might not be an adequate surrogate endpoint in further clinical studies of CRLM.

Subset analysis showed that, among patients with fewer metastases (1-4), the CET group had better survival than the BEV group (HR: $0.260,95 \% \mathrm{Cl}$ : 0.084-0.811) but conversely among patients with $\geq 5$ metastases. Our inclusion criteria required that patients with $\geq 5$ metastases had tumours $\geq 5 \mathrm{~cm}$ in size. Therefore, the presence of fewer but larger metastases might be a good indication for the use of anti-EGFR antibody. Resection after shrinkage of a large tumour is the ideal clinical course for CETtreated patients. The New EPOC trial could not show any benefit of adding CET to standard chemotherapy in patients with resectable CRLM. ${ }^{20}$ However, this may be because CET is not effective for small resectable liver metastases but seems suitable for patients with fewer but larger metastases. 
$\mathbf{a}_{40}$

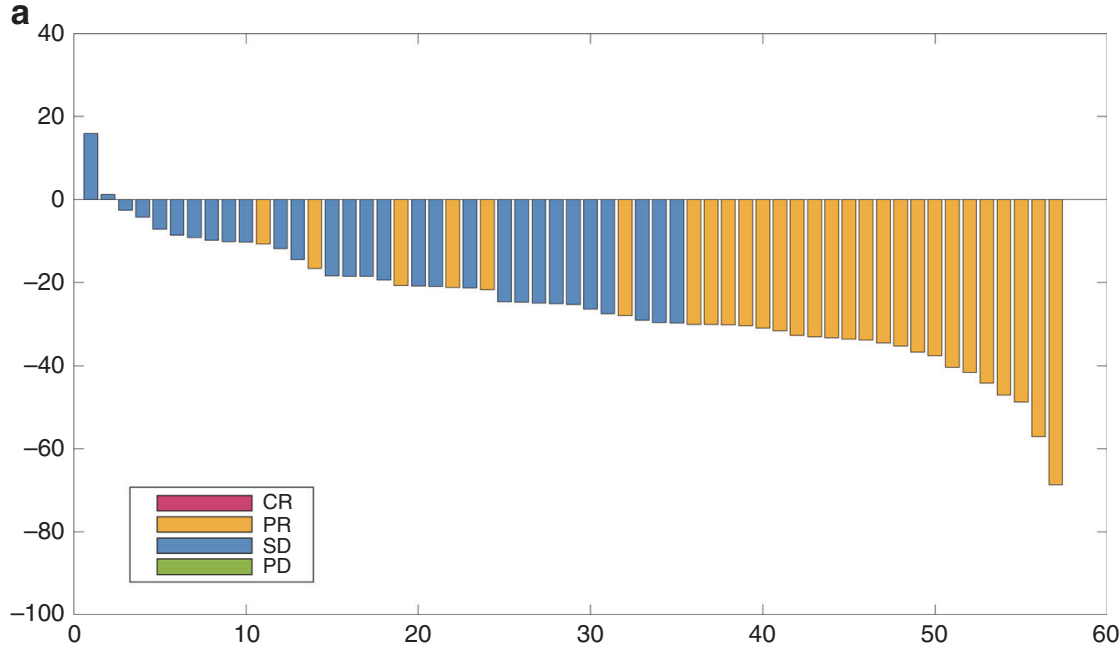

b

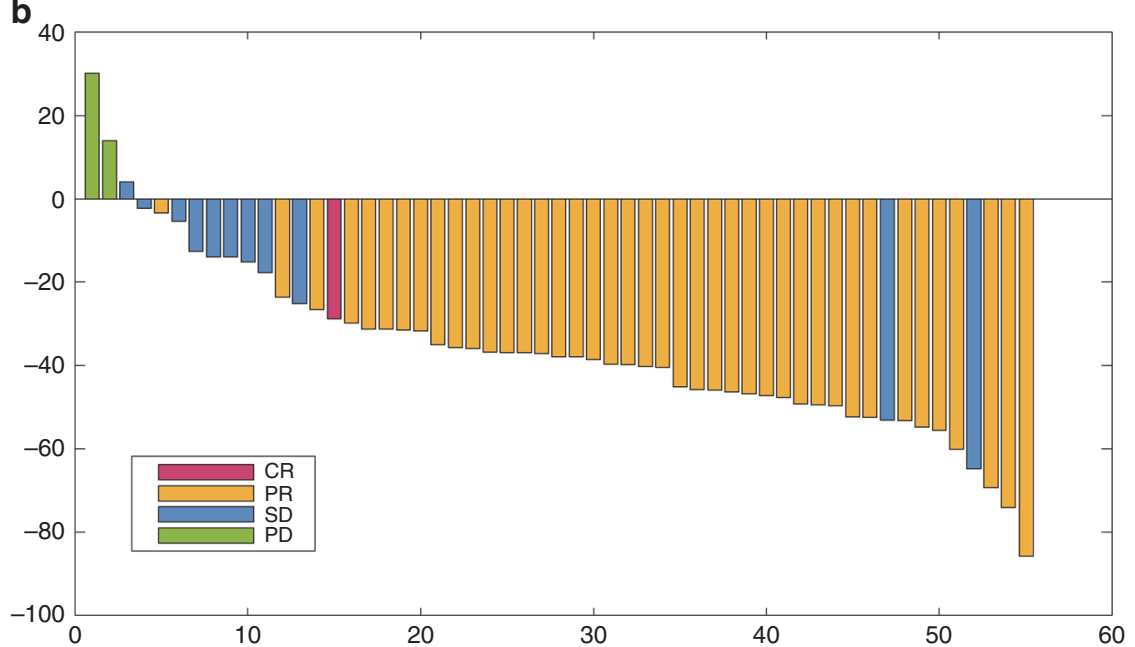

Fig. 4 Waterfall plot shows the best change in target lesion size for individual patients by central assessment. CR complete response, NE not evaluable, PD progressive disease, PR partial response, SD stable disease. a mFOLFOX6+bevacizumab and b mFOLFOX6+cetuximab

Tumour sidedness is also an important characteristic of metastatic CRC. Although previous studies indicated no survival benefit for CET in metastatic right-sided CRC, ${ }^{21}$ the PFS of patients with right-sided tumours in our trial showed similar or better results with CET as those with left-sided tumours. CET reportedly reduces tumour size better than $\mathrm{BEV}$, even in right-sided colon cancer. ${ }^{22}$ Therefore, in cases with metastatic CRC limited to the liver, sidedness might not be relevant for PFS, as half of the metastatic site will be surgically resected.

A previous study reported a higher pathological response rate for BEV than for CET. ${ }^{23}$ In contrast, the present study showed a higher pathological response rate for CET than for BEV, which had some effect on the survival of the patients who underwent surgery. In fact, the median PFS of patients with surgical conversion was 13.8 months ( $95 \% \mathrm{Cl}$ : 8.4-not reached) in the CET arm and 6.5 months (95\% Cl: 4.0-13.6) in the BEV arm (Supplementary Fig. S3). This difference will have some impact on final overall survival. We will investigate tumour regression grade (TRG), modified TRG, dangerous halo, and morphological response as exploratory endpoints. These analyses will elucidate as to what is the best pathological assessment of prognosis for CRLM.

The present trial was limited by its small sample size, and so the statistical power was insufficient to enable the comparison of the two biologic agents. Furthermore, the OS is immature and needs longer-term follow-up. In addition, the inclusion criteria for initially unresectable CRLM are controversial. We followed the criteria used in previous studies, which included patients with CRLM with $<5$ metastases but with tumour(s) $>5 \mathrm{~cm}$ in diameter. ${ }^{11,12}$ Some of these cases might have been resectable without chemotherapy, and this may have confounded the results.

In conclusion, CET showed a higher response rate than BEV among patients with initially unresectable liver-limited metastases and resulted in good PFS, especially for patients with fewer but larger metastases. However, there were no significant differences between BEV and CET in efficacy regarding resection rate, and both biologic agents had acceptable safety profiles. Both agents are viable treatment options for liver metastases with suboptimal resectability.

\section{ACKNOWLEDGEMENTS}

We thank all the patients, their families, and the investigators involved in this study. We also thank Marla Brunker and Kelly Zammit from Edanz Group (www. edanzediting.com/ac) for editing a draft of this manuscript.

\section{AUTHOR CONTRIBUTIONS}

Y.E., E.O., K.M., T.Y., and Y.M. participated in the study conception and design. E.O., Y.E., H.U., K.M., T.T., T.N., E.H., H.O., D.M., T.K., Y.K., T.F. and K.Y. provided study materials or recruited patients. E.O., Y.E., T.Y., H.U., K.M., M.U., I.H., N.T., K.S. and Y.M. were 
involved in data analysis and interpretation and writing and development of the manuscript. All authors approved final draft.

\section{ADDITIONAL INFORMATION \\ Supplementary information is available for this paper at https://doi.org/10.1038/} s41416-019-0518-2.

Competing interests: E.O. received grants from Chugai Pharmaceutical Co., Ltd., and received lecture fee from Chugai Pharmaceutical Co., Ltd., Taiho Pharmaceutical Co., Ltd., Eli Lilly, Bayer Japan, Yakult Honsha Co., Ltd., Merck Serono, and Takeda Pharmaceutical Co., Ltd. Y.E. received honoraria from Chugai Pharmaceutical Co., Ltd. T.Y. reports grants and received lecture fee from Chugai, Takeda, and Taiho and grants from Pfizer, Merck Serono, Astra Zeneca, Yakult Honsha, Bristol Myers Squibb, Daiichi-Sankyo, Gilead Sciences, Huya Biosciences, Bayer, and Sysmex. H.U. reports grants from Taiho Pharm., Chugai Pharm., Takeda Pharm., and Yakult. K.M. received grants from Chugai, MSD, Daiichi Sankyo, Shionogi, Kyowa Hakko Kirin, Gilead Sciences, Sanofi, Pfizer and Merck Serono, and personal fees from Taiho, Takeda, Bayer, and Eli Lilly. T.T. received lecture fees from Chugai Pharmaceutical Co., Ltd., Takeda Pharm,Sanofi, Taiho Pharm, Merck Serono, and Yakult Honsha. T.F. received grants from Astellas Pharma Inc., Otsuka Pharmaceutical Co., Sanofi, Taiho Pharmaceutical, Takeda Pharmaceutical Co., Chugai Pharmaceutical Co., Eli Lilly Japan K.K., and Yakult Honsha Co. K.Y. received grants and personal fees from Chugai Pharm. Co., Ltd., Merck Serono Co., Ltd., and Yakult Honsha Co., Ltd., grants from Kyowa Hakko Kirin, grants and personal fees from Nippon Kayaku Co., Ltd. during the conduct of the study, grants and personal fees from Johnson \& Johnson K.K., Taiho Pharm. Co., Ltd., Daiichi Sankyo Co., Ltd., Tsumura, Takeda Pharmaceutical Co., Ltd., Asahi Kasei, Eisai, Sanofi, Eli Lilly Japan K.K., Covidient Japan, Novartis Pharma, EA Pharma Co., Ltd. and Ono Pharm. Co., Ltd, grants from Toyama Chemical, Abbott Japan, Otsuka Pharma Co., Ltd., Toray Medical Co., Ltd, KCl, Astellas, Sumitomo Dainippon Pharma, and Bristol Myers Japan and personal fees from Olympus, Terumo, Denka, MSD K.K., Bayer Yakuhin, Ltd., and Intuitive Surgical. M.U. received grants from Chugai, Yakult Honsha, and Takeda and received personal fees from Merck Serono, Chugai, Yakult Honsha, and Takeda during the conduct of the study, grants from Taiho, Novartis, Asahi Kasei, Asteras, Toyama Chem, and Pfizer and personal fees from Taiho, Novatis, Asahi Kasei, Pfizer, Teijin Pharm, Eisai, Daiichi Sankyo, Johnson \& Johnson, Boston Scientific, Covidien/Medtronic, Nobelpharma, Ono Pharmaceutical, and Mylan. I.H. reports grants and personal fees from Taiho Pharma, Chugai Pharma, Daiichi-Sankyo Pharma, Yakult-Honsha Pharma, Lilly, Takeda Pharma, and Ono Pharma. N.T. received grants from Taiho Pharmaceutical Co. and Chugai Pharmaceutical Co., outside the submitted work. K. S. received honoraria from Chugai Pharm, Taiho, Takeda, Merck Serono, Yakult Honsha, Bristol-Meyers Japan, Bayer Yakuhin, and Eli Lilly Co. and received grants from Chugai, Taiho and Takeda. Y.M. received grants from Chugai Pharmaceutical Co., Ltd., Yakult Honsha Co., Ltd., and Merck Serono Co. Ltd. during the conduct of the study and grants from Taiho Pharmaceutical Co., Ltd., Ono Pharmaceutical Co., Ltd., Eli Lilly Japan K.K., Otsuka Pharmaceutical Co., Ltd., DAllCHI SANKYO COMPANY, LIMITED., Pfizer Japan Inc., Astellas Pharma Inc., Sumitomo Dainippon Pharma Co., Ltd., Eisai Co., Ltd., Shionogi \& Co., Ltd., and Novartis Pharma K.K. The other authors declare no competing interests.

Ethics approval and consent to participate: All patients provided informed consent (IC) to enter the study at the time of enrolment. The study was conducted in compliance with the principles of the Declaration of Helsinki, the international conference on harmonisation of technical requirements for registration of pharmaceuticals for human use guidelines and local ethical and legal requirements. The protocol and IC document were approved by the independent institutional review boards of all participating institutions. The names of all institutions are listed in the Supplementary Data.

Funding: This work was supported by EPS Corporation, with research contract funding from Chugai Pharmaceutical Co. Ltd, Japan.

Data availability: The data sets used and/or analysed during the current study are available from the corresponding author on reasonable request.

Publisher's note: Springer Nature remains neutral with regard to jurisdictional claims in published maps and institutional affiliations.

\section{REFERENCES}

1. Ferlay, J., Soerjomataram, I., Dikshit, R., Eser, S., Mathers, C., Rebelo, M. et al. Cancer incidence and mortality worldwide: sources, methods and major patterns in GLOBOCAN 2012. Int. J. Cancer 136, E359-E386 (2015).
2. Kato, T., Yasui, K., Hirai, T., Kanemitsu, Y., Mori, T., Sugihara, K. et al. Therapeutic results for hepatic metastasis of colorectal cancer with special reference to effectiveness of hepatectomy: analysis of prognostic factors for 763 cases recorded at 18 institutions. Dis. Colon Rectum 46(10 Suppl), S22-S31 (2003).

3. Cancer statistics in JAPAN-2016. https://ganjoho.jp/reg stat/statistics/brochure/ backnumber/2016_jp.html (2018).

4. Adam, R., Delvart, V., Pascal, G., Valeanu, A., Castaing, D., Azoulay, D. et al. Rescue surgery for unresectable colorectal liver metastases downstaged by chemotherapy: a model to predict long-term survival. Ann. Surg. 240, 644-657 (2004).

5. Folprecht, G., Grothey, A., Alberts, S., Raab, H. R. \& Kohne, C. H. Neoadjuvant treatment of unresectable colorectal liver metastases: correlation between tumour response and resection rates. Ann. Oncol. 16, 1311-1319 (2005).

6. Alberts, S. R., Horvath, W. L., Sternfeld, W. C., Goldberg, R. M., Mahoney, M. R., Dakhil, S. R. et al. Oxaliplatin, fluorouracil, and leucovorin for patients with unresectable liver-only metastases from colorectal cancer: a North Central Cancer Treatment Group phase II study. J. Clin. Oncol. 23, 9243-9249 (2005).

7. Folprecht, G., Gruenberger, T., Bechstein, W. O., Raab, H. R., Lordick, F., Hartmann, J. T. et al. Tumour response and secondary resectability of colorectal liver metastases following neoadjuvant chemotherapy with cetuximab: the CELIM randomised phase 2 trial. Lancet Oncol. 11, 38-47 (2010).

8. Garufi, C., Torsello, A., Tumolo, S., Ettorre, G. M., Zeuli, M., Campanella, C. et al. Cetuximab plus chronomodulated irinotecan, 5-fluorouracil, leucovorin and oxaliplatin as neoadjuvant chemotherapy in colorectal liver metastases: POCHER trial. Br. J. Cancer 103, 1542-1547 (2010).

9. Gruenberger, T., Bridgewater, J., Chau, I., Garcia Alfonso, P., Rivoire, M., Mudan, S. et al. Bevacizumab plus mFOLFOX-6 or FOLFOXIRI in patients with initially unresectable liver metastases from colorectal cancer: the OLIVIA multinational randomised phase II trial. Ann. Oncol. 26, 702-708 (2015).

10. Wong, R., Cunningham, D., Barbachano, Y., Saffery, C., Valle, J., Hickish, T. et al. A multicentre study of capecitabine, oxaliplatin plus bevacizumab as perioperative treatment of patients with poor-risk colorectal liver-only metastases not selected for upfront resection. Ann. Oncol. 22, 2042-2048 (2011).

11. Oki, E., Emi, Y., Miyamoto, Y., Kabashima, A., Higashi, H., Ogata, Y. et al. Phase II trial of S-1 and oxaliplatin plus cetuximab for colorectal cancer patients with initially unresectable or not optimally resectable liver metastases (KSCC1002). Ann. Surg. Oncol. 22(Suppl 3), S1067-S1074 (2015).

12. Beppu, T., Emi, Y., Tokunaga, S., Oki, E., Shirabe, K., Ueno, S. et al. Liver resectability of advanced liver-limited colorectal liver metastases following $\mathrm{mFOL-}$ FOX6 with bevacizumab (KSCC0802 Study). Anticancer Res. 34, 6655-6662 (2014).

13. Shindoh, J., Loyer, E. M., Kopetz, S., Boonsirikamchai, P., Maru, D. M., Chun, Y. S. et al. Optimal morphologic response to preoperative chemotherapy: an alternate outcome end point before resection of hepatic colorectal metastases. J. Clin. Oncol. 30, 4566-4572 (2012).

14. Hatano, E., Okuno, M., Nakamura, K., Ishii, T., Seo, S., Taura, K. et al. Conversion to complete resection with mFOLFOX6 with bevacizumab or cetuximab based on Kras status for unresectable colorectal liver metastasis (BECK study). J. Hepatobiliary Pancreat. Sci. 22, 634-645 (2015).

15. Stintzing, S., Modest, D. P., Rossius, L., Lerch, M. M., von Weikersthal, L. F., Decker, T. et al. FOLFIRI plus cetuximab versus FOLFIRI plus bevacizumab for metastatic colorectal cancer (FIRE-3): a post-hoc analysis of tumour dynamics in the final RAS wild-type subgroup of this randomised open-label phase 3 trial. Lancet Oncol. 17, 1426-1434 (2016).

16. Van Cutsem, E., Kohne, C. H., Hitre, E., Zaluski, J., Chang Chien, C. R., Makhson, A. et al. Cetuximab and chemotherapy as initial treatment for metastatic colorectal cancer. N. Engl. J. Med. 360, 1408-1417 (2009).

17. Schwartzberg, L. S., Rivera, F., Karthaus, M., Fasola, G., Canon, J. L., Hecht, J. R. et al. PEAK: a randomized, multicenter phase II study of panitumumab plus modified fluorouracil, leucovorin, and oxaliplatin (mFOLFOX6) or bevacizumab plus mFOLFOX6 in patients with previously untreated, unresectable, wild-type KRAS exon 2 metastatic colorectal cancer. J. Clin. Oncol. 32, 2240-2247 (2014).

18. Heinemann, V., von Weikersthal, L. F., Decker, T., Kiani, A., Vehling-Kaiser, U., Al-Batran, S. E. et al. FOLFIRI plus cetuximab versus FOLFIRI plus bevacizumab as first-line treatment for patients with metastatic colorectal cancer (FIRE-3): a randomised, open-label, phase 3 trial. Lancet Oncol. 15, 1065-1075 (2014).

19. Venook, A. P., Niedzwiecki, D., Lenz, H. J., Innocenti, F., Fruth, B., Meyerhardt, J. A. et al. Effect of first-line chemotherapy combined with cetuximab or bevacizumab on overall survival in patients with KRAS wild-type advanced or metastatic colorectal cancer: a randomized clinical trial. JAMA 317, 2392-2401 (2017). 
Randomised phase II trial of mFOLFOX6 plus bevacizumab versus mFOLFOX6... E Oki et al.

20. Primrose, J., Falk, S., Finch-Jones, M., Valle, J., O'Reilly, D., Siriwardena, A. et al. Systemic chemotherapy with or without cetuximab in patients with resectable colorectal liver metastasis: the New EPOC randomised controlled trial. Lancet Oncol. 15, 601-611 (2014).

21. Ciombor, K. K. \& Goldberg, R. M. Highlights in gastrointestinal (colorectal) cancer treatment: the primary tumor sidedness debate and advances in immunotherapy. JAMA Oncol. 2, 1537-1538 (2016).

22. Sunakawa, Y., Tsuji, A., Fujii, M. \& Ichikawa, W. No benefit from the addition of anti-EGFR antibody in all right-sided metastatic colorectal cancer? Ann. Oncol. 28, 2030-2031 (2017).

23. Ribero, D., Wang, H., Donadon, M., Zorzi, D., Thomas, M. B., Eng, C. et al. Bevacizumab improves pathologic response and protects against hepatic injury in patients treated with oxaliplatin-based chemotherapy for colorectal liver metastases. Cancer 110, 2761-2767 (2007).
Open Access This article is licensed under a Creative Commons Attribution 4.0 International License, which permits use, sharing, adaptation, distribution and reproduction in any medium or format, as long as you give appropriate credit to the original author(s) and the source, provide a link to the Creative Commons license, and indicate if changes were made. The images or other third party material in this article are included in the article's Creative Commons license, unless indicated otherwise in a credit line to the material. If material is not included in the article's Creative Commons license and your intended use is not permitted by statutory regulation or exceeds the permitted use, you will need to obtain permission directly from the copyright holder. To view a copy of this license, visit http://creativecommons. org/licenses/by/4.0/.

(c) The Author(s) 2019 